\title{
KOMPETENZZENTRUM
}

\section{Informationen sammeln und vermitteln}

\author{
Juliane Meinhold
}

Das Kompetenzzentrum Persönliches Budget des Paritätischen Wohlfahrtsverbandes begleitet mit Unterstützung des Bundesministeriums für Arbeit und Soziales die Umsetzung der neuen Leistungsform. Denn die Achillesferse des neuen Angebots stellt nach wie vor die oft als unzureichend empfundene Information aller Beteiligten dar: der Menschen mit einer Behinderung und ihren Angehörigen, der Leistungsträger und der Leistungsanbieter.

»Können Sie mir etwas zum Persönlichen Budget sagen?« So lautet die meistgestellte Frage am Beratungstelefon des Kompetenzzentrums Persönliches Budget des Paritätischen Wohlfahrtsverbandes. Bis zu 15mal täglich wird diese für die verschiedenen relevanten Akteure im Rahmen des Trägerübergreifenden Persönlichen Budgets beantwortet: für Menschen mit Behinderungen, für deren Angehörige, für Leistungserbringer und auch für Rehabilitationsträger.

Seit dem 1. Januar 2008 arbeitet das Kompetenzzentrum Persönliches Budget in Berlin in der Trägerschaft des Paritätischen Wohlfahrtsverbandes - Gesamtverband e. V. zusammen mit den Landesverbänden des Paritätischen und wird vom Bundesministerium für Arbeit und Soziales gefördert. Bis 2010 wird der Umsetzungsprozess des Trägerübergreifenden Persönlichen Budgets bundesweit durch das Kompetenzzentrum unterstützt und kritisch begleitet.

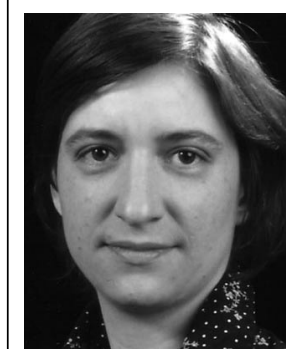

Juliane Meinhold ist Referentin im Kompetenzzentrum Persönliches Budget des Paritätischen Wohlfahrtsverbandes in Berlin. Die Diplom-Juristin mit einem Bachelor in Sozialwissenschaften ist seit 2004 in der Behindertenhilfe und der psychosozialen Versorgung in Berlin tätig.

E-Mail juliane.meinhold@paritaet.org

Diskussionen und Klärung offener Fragen nach wie vor groß ist.

Denn ungeachtet der Einführung des Persönlichen Budgets bereits im Jahre 2001 in das Sozialgesetzbuch Neuntes Buch (SGB IX - Rehabilitation und Teilhabe behinderter Menschen), einer dreijährigen Modellphase mit 14 Modellregionen in Deutschland von 2004 bis 2007 und trotz Inkrafttreten des Rechtsanspruches zum 1. Januar 2008 harren viele Details noch der Klärung. Allgemeinverbindliche Aussagen zur Umsetzung des Trägerübergreifenden Persönlichen Budgets treffen derzeit § 17 SGB IX (i. V. m. § 159 Abs.5 SGB IX) und die Budgetverordnung in Verbindung mit den sonstigen binden"Pauschalen widersprechen der Idee
des Persönlichen Budgets" den Vorschriften des Sozial(leistungs)rechtes. Sowohl die Handlungsempfehlung der Bundesarbeitsgemeinschaft für Rehabilitation (BAR) als auch die Handlungsempfehlung der Bundesagentur für

Die Aufgaben des Kompetenzzentrums sind neben den telefonischen Erstberatungen die Durchführung von insgesamt 16 Regionalkonferenzen in den Bundesländern zum Persönlichen Budget. Während der Regionalkonferenzen kommen Vertreter der am Persönlichen Budget beteiligten Akteure zusammen und erhalten eine Plattform für aktuelle Informationen regionaler sowie übergreifender Art, für Diskussionen und die Möglichkeit der Vernetzung zum Thema Persönliches Budget. Insbesondere kommen Budgetnehmerinnen und Budgetnehmer selbst zu Wort, die über ihre praktischen Erfahrungen mit dem Persönlichen Budget berichten. Leistungserbringer und Rehabilitationsträger zeigen ihre Erfahrungen und Perspektiven zur Umsetzung der neuen Leistungsform auf. Die drei ersten Regionalkonferenzen in Lübeck, Rostock und Halle mit durchschnittlich 200 Teilnehmenden haben deutlich gemacht, dass der Bedarf an Informationen, Arbeit geben, wie der Name schon sagt, Empfehlungen für die beteiligten Kostenträger, wie ein Persönliches Budget umzusetzen ist. Allerdings konnten für die Erarbeitung der Handlungsempfehlung der Bundesarbeitsgemeinschaft für Rehabilitation wichtige Partner wie die örtlichen Sozialhilfe- und Kinder- und Jugendhilfeträger nicht gewonnen werden. Dort erfolgt also sowohl eine kommunal- wie kostenträgerspezifische Umsetzung der Persönlichen Budgets. Für Leistungserbringer bedeutet dies, flexibel mit Angeboten auf unterschiedlich ausgestaltete Persönliche Budgets zu reagieren.

Die Abweichungen in der Ausgestaltung zeigen sich an unterschiedlichen Stellen. Neben der Anwendung einer Vielzahl differierender Hilfebedarfsfeststellungsverfahren allein bei den Sozialhilfeträgern und zusätzlich zwischen den Kostenträgern sind insbesondere die Differenzen in 
der Festlegung der Budgethöhen für Leistungsanbieter relevant. So werden derzeit beispielsweise in Sachsen-Anhalt in der Regel Budgetpauschalen der Bemessung der Budgethöhen zugrunde gelegt. Es erfolgt eine Einstufung der Budgetnehmerinnen und Budgetnehmer in vier Kategorien von Behinderungen (geistig behinderte Menschen, seelisch behinderte Menschen, seelisch behinderte Menschen infolge von Sucht, körperbehinderte Menschen) und in vier mögliche Hilfebedarfsgruppen. Jede Hilfebedarfsgruppe jeder Kategorie der Behinderung ist mit einer Pauschale unterlegt. Diese reichen von mindestens 271 Euro bis maximal 816 Euro pro Budget pro Monat. Damit wird dem in §17 SGB IX als Maßgabe festgelegten individuell festzustellenden Bedarf nicht Rechnung getragen. In vielen Fällen ist fraglich, ob die pauschalen Budgethöhen für die Deckung des individuellen Bedarfs ausreichend sind. In anderen Bundesländern oder Regionen werden dagegen Stundensätze unterschieden nach Fachleistungsstunden und Nichtfachleistungsstunden zur Budgetbemessung angesetzt. Auch hier gibt es keine einheitlichen Werte sondern regionale Unterschiede. Im schlechtesten Falle werden pro Nichtfachleistungsstunde vonseiten des Kostenträgers beispielsweise 6,55 Euro festgelegt (so Sozialagentur Sachsen-Anhalt für eine Pflegeassistenz). Gerichtlich konnten letztlich 9,06 Euro pro Stunde durchgesetzt werden (Sozialgericht Halle AZ: S 16 KR 263/07 ER, 18.12.2007).

Die in der Zielvereinbarung zwischen Rehabilitationsträger und Budgetnehmenden getroffenen Festlegungen geben Anhaltspunkte für die erforderlichen Hilfen und bilden für die Budgetnehmerinnen und Budgetnehmer - und letztlich für den Leistungsanbieter - die Grundlage für die monatliche Umsetzung der zur Verfügung stehenden Budgets. An dieser Stelle entstehen jedoch weitere Fragen für Leistungserbringer hinsichtlich der Ausgestaltung und Kalkulation der Angebote für Budgetnehmerinnen und Budgetnehmer, hinsichtlich der Personalverwaltung, des Marketings und vieles mehr.

Das Kompetenzzentrum Persönliches Budget des Paritätischen Wohlfahrtsverbandes verfolgt diese Entwicklungen und Fragestellungen, sammelt entsprechende Informationen und stellt sie auf einer eigenen Internetseite zur Verfügung (www.budget.paritaet.org). Leistungsanbieter können sich hier informieren, Anregungen für eigene Ideen und Projekte einholen, Veranstaltungs- und Weiterbildungstipps bekommen, weitere Ansprechpartner für Fragen finden oder sich als solche zur Verfügung stellen. Ein Beispiel für die Informationsarbeit des Kompetenzzentrums ist das permanent aktualisierte Verzeichnis der Beratungsstellen zum Persönlichen Budget auf der erwähnten Internetseite. Über das Verzeichnis zeigen neben Institutionen und Gruppen der Selbsthilfe auch Leistungsanbieter Beratungsmöglichkeiten zum Persönlichen Budget an. Nach Postleitzahlen geordnet ermöglicht es mit derzeit über 120 Angeboten einen guten Überblick zu den Beratungsmöglichkeiten in den unterschiedlichen Regionen in Deutschland.

Die Vernetzung der registrierten Beratungsstellen ist eine der weiteren Aufgaben des Kompetenzzentrums. Der Austausch über die Beratungstätigkeit soll befördert und die Diskussion gemeinsamer Standards für die Beratungstätigkeit angeregt werden - eine anspruchsvolle Aufgabe angesichts der Vielfältigkeit der Beratungslandschaft. Gleichzeitig eine wichtige Aufgabe, denn eine auf lange
Sicht angelegte, kompetente und umfassende Beratung für Menschen mit Behinderungen und deren Angehörige ist der sicherste Zugang zum Persönlichen Budget - und momentan nach wie vor ein wunder Punkt.

In der Regel werden die Beratungsstellen mit einer umfassenden Spanne an Fragen zur Lebens- und Zukunftsplanung, zum gesamten Leistungsspektrum für Menschen mit Behinderungen und zu einzelnen Leistungsvoraussetzungen konfrontiert. Bezüglich dieser Anforderungen müssen und wollen viele Beratungsstellen ihre Kompetenzen ausbauen. Darüber hinaus werden viele Beratungsstellen ausschließlich über Zuwendungen für bestimmte Zeiträume gefördert, andere beruhen auf der Selbstinitiative von unabhängigen Gruppen und Leistungsanbietern. Nur einige Landesverbände und kommunale Träger zeigen sich engagiert und nehmen ihre Beratungspflichten ernst. Das installierte Netz an Servicestellen dagegen zeigt bis auf wenige Ausnahmen eine unzureichende Beratungskompetenz zum Persönlichen Budget.

Dies macht deutlich, dass die registrierten Beratungsmöglichkeiten kein dauerhaft gesichertes, gesteuertes und infrastrukturell gut verteiltes Angebot für Menschen mit Behinderungen und deren Beratungs- und Unterstützungsbedarf in Fragen des Persönlichen Budgets darstellt, sondern von gegebenen Beratungskompetenzen, begrenzten Zuwendungsfinanzierungen und dem guten Willen der Beratungsanbieter abhängt.

Angesichts dieser Herausforderungen in der Umsetzung des Trägerübergreifenden Persönlichen Budgets als wichtiges Instrument für die Stärkung der Selbstbestimmung der Menschen mit Behinderungen wird das Kompetenzzentrum Persönliches Budget des Paritätischen bei der Suche nach fachlich angemessenen und wirtschaftlich trägfähigen Antworten behilflich sein.

Das Kompetenzzentrum Persönliches Budget des Paritätischen Wohlfahrtsverbandes bietet:

- Erstberatung und Vermittlung zu Fragen des Persönlichen Budgets

- aktuelle Informationen und grundlegende Ausführungen zum Persönlichen Budget über eine eigene Website (www.budget.paritaet.org)

- regionale und bundesweite Fachtagungen zur Information und Diskussion aktueller Themen zum Persönlichen Budget

- Unterstützung bei der Vernetzung von Beratungsstellen

- Erstellung von Informationsmaterialien zum Persönlichen Budget

Kompetenzzentrum Persönliches Budget des Paritätischen

Oranienburger Straße 13-14, 10178 Berlin

Telefon 03024533170

Fax 03024636110

E-Mail budget@paritaet.org

Internet http://www.budget.paritaet.org

Ansprechpartnerin: Juliane Meinhold 